\title{
Valuation of European Call Options Using Wavelet-Based Pricing Model and Black-Scholes Pricing Model
}

\author{
Sigei Sheila Chepkorir, Anthony Gichuhi Waititu, Jane Aduda Akinyi \\ Department of Statistics and Actuarial Sciences, Jomo Kenyatta University of Agriculture and Technology, Nairobi, Kenya \\ Email address: \\ shylachepkorir2@gmail.com (S. S. Chepkorir), agwaititu@gmail.com (A. G. Waititu),jaduda@jkuat.ac.ke(J. A. Akinyi)

\section{To cite this article:} \\ Sigei Sheila Chepkorir, Anthony Gichuhi Waititu, Jane Aduda Akinyi. Valuation of European Call Options Using Wavelet-Based Pricing \\ Model and Black-Scholes Pricing Model. International Journal of Data Science and Analysis. Vol. 5, No. 5, 2019, pp. 91-98. \\ doi: $10.11648 / j . i j d s a .20190505 .13$
}

Received: October 2, 2019; Accepted: October 12, 2019; Published: October 28, 2019

\begin{abstract}
The Black- Scholes model is a well-known model for hedging and pricing derivative securities. However, it exhibits some systematic biases or unrealistic assumptions like the log-normality of asset returns and constant volatility. A number of studies have attempted to reduce these biases in different ways. The objective of this study is to value a European call option using a non-parametric model and a parametric model. Amongst the non-parametric approaches used to improve the accuracy of the model in this study is the Wavelet-based pricing model. This model is found as promising alternative as far as pricing of European options is concerned, due to its varied volatility of the underlying security and estimation of the risk neutral MGF. This study made an attempt to improve the accuracy of option price estimation using Wavelet method and it improves the accuracy due to its ability to estimate the risk neutral MGF. The MSE and RMSE of Wavelet model is 0.208546 and 0.456669 respectively which is much lower than that of Black-Scholes model and therefore in conclusion, Wavelet model outperforms the other model. The study was carried out using simulated stock prices of 1024 observations.
\end{abstract}

Keywords: Options, Wavelet Model, Derivatives, MGF

\section{Introduction}

The financial contracts or instruments which derive their value from some other variables are called Derivatives. Derivatives are instruments whose value depend on an underlying asset. Equity, commodity, bond or currency, stocks, interest rate, exchange rate or any other financial variables of interest to the researcher could be the underlying asset. Derivatives includes; Forwards, futures, options and swaps. These derivatives can either be exchange- traded or traded over the counter (OTC). The ones traded on the exchange are standardized and regulated.

An option is a type of derivative that gives the option holder the right but not obligation to buy or sell an underlying security at a specified price, at any time on or before a given date in the future as agreed on. According to the right to sell or to buy, we distinguish the two types of options. The holder of a call option has the right but not obligation to buy the underlying security at a specified price, at a specified time in the future. The holder of a put option has the right but not the obligation to sell underlying security under some predefined terms of an agreement which includes; strike or exercise price, maturity date and option volume. European-style options can be exercised at the maturity date only while American options can be exercised at any time prior to or on the maturity date. American options need more complex pricing methodology due to the extra feature of early exercising. The Monte Carlo method, which is based on repeated computation and random sampling can be used for pricing American options [1].

In the Kenyan market, derivatives are yet to be developed. The derivatives market has been inactive due to some of the factors such as low level of investor awareness and sophistication, inadequate risk management, lack of commodities on large scale and inadequate liquidity. This study therefore prices a European option using two nonparametric methods and a parametric method.

The Black-Scholes option pricing formula which has been used as the benchmark to price European options in most of the previous researches due to its simplicity and low computational demand [2]. This Black-Scholes formula remains the most widely used model in pricing options 
though it has some known biases which include volatility smiles and skewness. The Black Scholes model has been developed under some assumptions such as the risk-free rate and volatility of the returns are known and constant, the returns of the underlying security are normally distributed, markets are efficient (market movements cannot be predicted).

In the real market, the asset returns follow a leptokurtic distribution which is in contrast to the Black-Scholes model where returns are assumed to be log-normally distributed. Also, in practice the volatility should vary in the market and as a result of the Black Scholes' assumption of constant volatility it results to volatility smiles. In the previous researches, it has been shown that distribution in the option prices is skewed to the left (negatively skewed) [3].

This model derives the closed form solution for pricing of a European options that is why it is used as a benchmark. Variables used in this model are observable, for example the time to expiration, exercise price and the closing price except the volatility which is not directly observable. The Black Scholes model therefore belongs to the parametric continuous time models with a closed form solution family.

The Wavelet based pricing model is another nonparametric method alternative used to price derivatives [4]. According to the literature, this model is the latest theoretical contribution to the option pricing and it is better at capturing the volatility smiles which is as a result of the Black-Scholes' assumption on volatility constant.

The implied risk neutral moment-generating functions (MGF) is estimated by the wavelet method. This method only requires a reasonable amount of data (different strikes) and is very efficient, unlike other non-parametric methods which requires large amount of data.

This research therefore compared the performance of Wavelet based pricing model and Black-Scholes model in the valuation of a European option.

The remainder of the paper is structured as follows: Section 2 discusses the existing literature; Section 3 presents an overview of the Black- Scholes model and Wavelet based pricing model. Section 4 describes the data, shows the empirical results and performance measures of the models. Lastly, section 5 concludes the study.

\section{Literature Review}

The simple closed form solution of European options was derived during the financial crisis [2]. Their formula can be used to obtain the following parameters; the spot price, the exercise price, interest rate, time to maturity and volatility. A lot of improvements have been done to the original BlackScholes formula since the paper of [2]. This model has been discussed extensively in order to improve its pricing biases and to impose more practical assumptions. The derivation of the pricing formula is independent on the parametric form of the underlying security's price dynamics and hence the success of this model depends on the accurate capture of dynamics of the underlying security's price process.
The Black Scholes model does not correctly price in high volatility markets [5]. For out-of-money puts, there is high implicit volatility relative to the at-the- money calls and puts [3].

According to the researches as far as pricing derivative securities is concerned, Wavelet based option pricing model is the latest option pricing model in the literature [6].

The approximation of the implied risk-neutral MGF of the underlying security (asset) returns was focused by developing a non -parametric option pricing model called Wavelet model [4]. In comparison between the risk-neutral MGF and the implied risk-neutral PDF, the risk-neutral MGF has a number of advantages even though between them, there is a one to one relationship. The following are some examples of these advantages; when there is presence of jumps in the underlying process MGF is more tractable, the option's obtained MGF is a continuous function, statistical moments of the asset distributions can be obtained using MGF and the risk neutral MGF can be used to estimate the out of sample options that has maturity dates which are different.

The following are some of the applications of wavelet method in finance and economics as pointed out in [6] and [7]; Wavelets can be used in multi-scaling analysis. For example, analyzed the relationship between economic variables at different scale by using the wavelet method and they found out that over a different time horizon, the relationship changes [8]. Employing the wavelet method to de-seasonalize prices of electricity [9].

The second use of wavelet method is that, they can be used to de-noise raw data. Also, wavelets can be used to improve analysis of volatility since they are a preprocessing denoising tool [10]. The de-noising ability of wavelets was also recognized in [7]. Evidence have been provided to support that wavelet based local linear approximation [11].

Algorithm has a superior performance in de-noising financial data with high frequency by using the first principal component to de-noise frequency variations of a business cycle with wavelets [12].

Lastly, wavelets can be used to estimate parameters of the models which are unknown using wavelets in pricing of an American derivative security by levy process [13]. European options can also be priced using the Shannon wavelet [14]. Another example is the application of the wavelets in nonparametrically estimation of a diffusion function [15]. Wavelets can also be used in calibrating parameters with long memory processes [16].

\section{Methodology}

\subsection{The Black-Scholes Model}

The Black and Scholes (1973) option pricing formula prices European style derivative security on stocks with no dividend paid. The following are some of the assumptions of Black-Scholes model;

i. The risk-free interest rate and volatility are known and 
constant through time.

ii. The options are European-style options.

iii. The returns on the underlying stock prices are lognormally distributed.

iv. Security trading is continuous.

v. No transaction costs or services with buying or selling the option.

The Black -Scholes formula is the solution of the BlackScholes partial differential equation under the boundary conditions $g=\max (0, S 0-K)$ for call and $g=\max (\mathrm{K}-S 0,0)$ for put when $\mathrm{t}=\mathrm{T}$. The Black-Scholes formula is then defined as;

$$
\begin{gathered}
c=S 0 N(d 1)-K \exp (-r T) N(d 2) \\
p=K \exp (-r T) N(-d 2)-S 0 N(d 1)
\end{gathered}
$$

Where;

$$
\begin{gathered}
d 1=\frac{\ln (S 0 / K)+\left(r+\sigma^{2} / 2\right) T}{\sigma \sqrt{ } T} \\
d 1=\frac{\ln (S 0 / K)+\left(r-\sigma^{2} / 2\right) T}{\sigma \sqrt{T}}=\mathrm{d} 1-\sigma \sqrt{T}
\end{gathered}
$$

From the Black-Scholes formula above, we see that the strike price $(K)$, risk free interest rate $(r)$, time to maturity $(T)$, the volatility $(\sigma)$ and the current price of the stock $(S 0)$ are required. $c$ is the call option price, $p$ is the put option price and $N($.$) is the cumulative distribution function.$

\subsection{Wavelet Based Pricing Model}

As it has been seen from the reviewed literature, this model is a new method in the field of finance. Based on some general assumptions like independent and identically distribution (iid) for asset returns and that the moment generating function is defined well, the wavelet based pricing model can be expressed as follows;

$$
V_{t}\left(S_{0} ; K ; T\right)=K e^{-r(T-t)} \alpha^{-1}\left(\frac{\Theta^{T-t}(v)}{v(v+1)}\right)\left(\ln \frac{S_{0}}{K}\right)
$$

Where, $t$ is the current time, $S_{0}$ is the underlying asset price at time $t, V_{t}$ is the time $t$ price for a European call option written on asset, $K$ is the strike price and $T$ is the future maturity date. $\mathcal{\delta}^{-1}$ is the bilateral inverse Laplace transform. Interest rate $r$ is assumed to be constant.

From the expression above, the core of the pricing model is; $\frac{\Theta^{T-t}(v)}{v(v+1)}$ where $v$ is a complex value whose real part $\operatorname{Re}(v)$ $<-1$ for calls and $\operatorname{Re}(v)>0$ for puts. The underlying asset dynamics and investor expectation in option prices is captured by the MGF $\Theta^{T-t}(v)$ of the logarithmic returns $\ln \frac{S_{0}}{K}$. When $T-t=1, \Theta(v)$ is the risk-neutral MGF for the rate of return per unit of time. This needs to be approximated by wavelets.

In order to approximate the implied MGF using the wavelet method, one has to choose a particular wavelet from a large family of wavelets. From the wavelet literature, researches have shown that there is no best wavelet for a particular application. And therefore, we choose a wavelet that can achieve a reasonable accuracy with wavelet terms being very minimal. On this criterion, the Franklin hat function performs very well because it is symmetric, smooth and piecewise continuous. This function also emulates the probability density function of asset returns.

To estimate the risk neutral MGF of the return per unit time, we use the Franklin hat function $f(t)$ expressed as;

$$
f(t)=\left\{\begin{array}{c}
(1-|t|), \text { if }-1 \leq t<1 \\
0, \text { Otherwise }
\end{array}\right.
$$

$m_{f}(\mathrm{v})$ denotes the Laplace transform of $\mathrm{h}(\mathrm{t})$, this is expressed as;

$$
m_{f}(v)=\left(\frac{e^{v / 2}-e^{-v / 2}}{v}\right)^{2}
$$

From the Franklin function $f(t)$, a set of generalized functions can be given as:

$$
f_{j, k}(t)=2^{\frac{j}{2}} h\left(2^{j} t-k\right), j, k=0, \pm 1, \pm 2, \ldots
$$

where $j$ is the scaling parameter which determines the degree of dilation, while $k$ is the shifting parameter that controls the horizontal location of the function.

Performing the Laplace transform on $f_{j, k}(t), m_{j, k}(v)$ is obtained as follows;

$$
m_{j, k}(v)=2^{\frac{-j}{v}} e^{\frac{-k v}{2^{j}}} m_{h}\left(\frac{v}{2^{j}}\right), j, k=0, \pm 1, \pm 2, \ldots
$$

From the set of the generalized Franklin hat function, the risk neutral MGF of the return $\Theta(v)$ can be expanded using the Laplace transform as follows:

$$
\Theta(v)=\sum_{j=-\infty}^{\infty} \sum_{k=-\infty}^{\infty} a_{j k} m_{j, k}(v)
$$

Where, $a_{j k}$ is a set of some unknown coefficients and this is estimated using the sum of squared error between the actual price and the estimated prices.

\subsection{Pricing Performance Measures}

In this study, for us to compare the pricing performance of the three models, we used the commonly used statistical criteria. These tests statistics include; Mean Squared Error (MSE) and Root Mean Squared Error (RMSE). They are expressed as;

$$
\begin{aligned}
M S E & =\frac{1}{n} \sum_{i=1}^{n}\left(y_{i}-\hat{y}_{i}\right)^{2} \\
R M S E & =\sqrt{\frac{1}{n} \sum_{i=1}^{n}\left(y_{i}-\hat{y}_{i}\right)^{2}}
\end{aligned}
$$

$n$ is the number of observations, $y_{i}$ is the estimated 
variable, $\hat{y}_{i}$ is the estimate of the variable. For MSE and RMSE, the smaller the values the better the model, that is lower values indicates a better fit or the closer the values are to zero, there is a perfect accuracy of the pricing performance.

\section{Data Description}

The data sets consist of simulated stock prices, the exercise price, the time to matureness, interest rate and volatility. In this study Monte Carlo simulation was used to generate 1024 stock prices. These data is divided into three, In-The-Money options, At-The-Money options and Out- of -The money options.

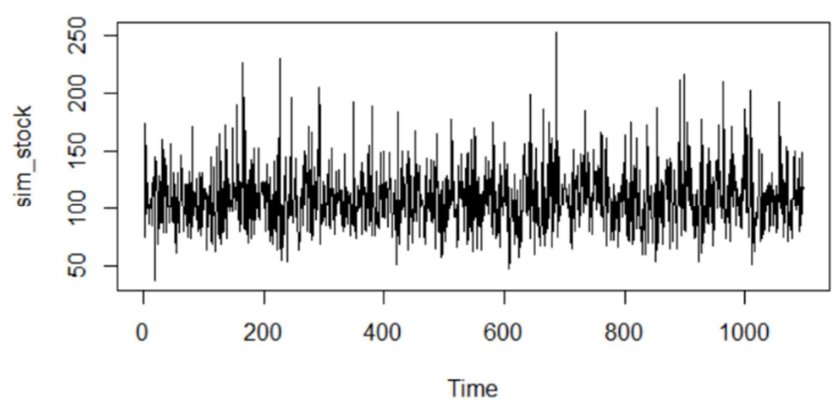

Figure 1. A plot of the simulated data.

Figure 1, is a time series plot of the simulated stock prices, and this plot displays the typical stylized facts present in the data.

\section{Empirical Result}

Value of Call and Put Option in Black-Scholes Model

In Black-Scholes Model, we estimate the value of a call and put option at different strike prices and time to maturity generated by simulation. In this study, we used the historical volatility. The current price $(S 0)$, historical volatility $(0.02626 \%)$ and interest rate $(7.020 \%)$ are kept constant.

Table 1. Value of call and put option in Black-Scholes Model with Time to Maturity of 1 year.

\begin{tabular}{lllll}
\hline Strike Price & $\mathbf{N}(\mathbf{d} 1)$ & $\mathbf{N}(\mathbf{d} 2)$ & $\begin{array}{l}\text { Value of } \\
\text { call option }\end{array}$ & $\begin{array}{l}\text { Value of put } \\
\text { option }\end{array}$ \\
\hline 90 & 0.49621 & 0.39958 & 16.10134 & $2.46363 * 10^{\wedge-12}$ \\
95 & 0.49621 & 0.39958 & 11.44035 & $5.56768^{*} 10^{\wedge-08}$ \\
100 & 0.49621 & 0.39958 & 6.78192 & 0.00266 \\
105 & 0.49621 & 0.39958 & 2.41484 & 0.29661 \\
110 & 0.49621 & 0.39958 & 0.23401 & 2.77060 \\
\hline
\end{tabular}

Table 2. Value of call and put option in Black-Scholes Model with Time to Maturity of 2 years.

\begin{tabular}{lllll}
\hline Strike Price & N (d1) & N (d2) & $\begin{array}{l}\text { Value of } \\
\text { call option }\end{array}$ & $\begin{array}{l}\text { Value of put } \\
\text { option }\end{array}$ \\
\hline 90 & 0.49621 & 0.39958 & 21.78905 & $5.43351^{*} 10^{\wedge-12}$ \\
95 & 0.49621 & 0.39958 & 17.44400 & $5.56768^{*} 10^{\wedge-08}$ \\
100 & 0.49621 & 0.39958 & 13.09091 & $5.39538^{*} 10^{\wedge-05}$ \\
105 & 0.49621 & 0.39958 & 8.76111 & 0.00722 \\
110 & 0.49621 & 0.39958 & 4.59987 & 0.19103 \\
\hline
\end{tabular}

From table 2, It can see that as the strike price increases the value of a call option decreases while the value of a put option increases. When the exercise price is lower, the higher the value of the call option. That is, the value of call option is inversely proportional to the strike price while the value of the put option is directly proportional to the strike price. This can be illustrated more by figure 2 .

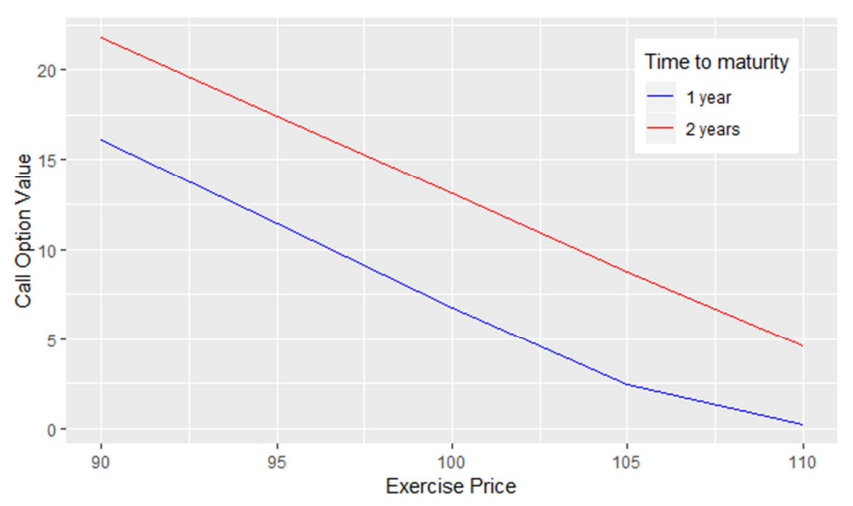

Figure 2. Exercise price against the Black-Scholes Call option value.

Table 1 and table 2 show the values of option with different time to maturities, it is seen that the value of the option is greater when there is more time to maturity. This is because the underlying asset has more potential for movement and hence the value of the option will be higher. For instance, when the strike price is 90 , the values of a call option are 16.10134 and 21.78905 with time to maturity of 1 year and 2 years respectively.

Value of a call option using Wavelet Based pricing model

Wavelet based pricing model has a shut structure answer for finding the cost of a European call option. In order to approximate the risk neutral MGF, the generated data set ( $\mathrm{S} 0$, $\mathrm{Ki}, \mathrm{T}, \mathrm{Ci}$ ) was used and wavelet analysis performed. The scale parameter and the shift parameter for the wavelet series chosen in this study is $\mathrm{j}=3$ and $\mathrm{k}=-4: 4$ respectively, this is because when the parameters increases, the computation time also increases and requires more data for optimization. The goals of the risk neutral MGF is determined by the scale parameter and therefore for the risk neutral MGF to be finer, scale parameter should be large given the shift parameter is chosen appropriately.

With an increment of 1 , the exercise values are evenly distributed from 90 and 100, the current price $S 0=100$, interest rate $(7.020 \%)$ and time to maturity (1 year) are the variables.

Our experiment started by first performing the decomposition of the simulated stock prices into various time scales utilizing the Haar mother wavelet. We carried out the wavelet decomposition of stock prices into a lot of 4 symmetrical components (D1, D2, D3, D4) that represent various parts of frequency and smoothed component $(s 4)$. This data deterioration permits time frequency domain of the original data. The level of decomposition was set to 4 levels because the wavelet coefficients gets smaller for higher level of decomposition. 
Table 3. Descriptive statistics of the decomposition levels.

\begin{tabular}{lllll}
\hline Statistic & D1 & D2 & D3 & D4 \\
\hline Mean & $-5.50080 * 10^{\wedge-15}$ & $-5.46508 * 10^{\wedge-15}$ & $-5.28873 * 10^{\wedge-15}$ & $-5.43141 * 10^{\wedge-15}$ \\
Median & 0.09199 & 0.01897 & 0.01091 & -0.00547 \\
Maximum & 6.33116 & 3.65259 & 2.83677 & 2.13669 \\
Minimum & -6.93500 & -3.95797 & -3.04179 & -2.23943 \\
Std. dev & 1.99486 & 1.35188 & 0.99491 & 0.75240 \\
Skewness & -0.07699 & 0.00588 & -0.00461 & -0.00968 \\
Kurtosis & 0.14562 & -0.27744 & -0.21892 & -0.13038 \\
\hline
\end{tabular}

The approximated risk-neutral MGF is equivalent to the sum of wavelet coefficients of the Franklin hat function. The value of European call options with various exercise prices and period to matureness is obtained by plugging in the approximated risk-neutral MGF in wavelet pricing formula. These estimated wavelet coefficients from the stock prices are; $-0.0342,0.2464,-0.0445,0.6407,-0.6842,0.4881$, $0.0240,-0.8679$ and 0.1246 . Many of these values are close to zero. It is found that, with only 9 Franklin hat function coefficients, we can estimate the risk-neutral MGF.

Table 4. Wavelet Decomposition Coefficients of different components (D1, $D 2, D 3, D 4)$.

\begin{tabular}{llll}
\hline D1 & D2 & D3 & D4 \\
\hline-0.36551 & 0.05374 & -0.06732 & 0.31609 \\
0.21675 & -0.03148 & -0.49820 & 0.76126 \\
0.10741 & -0.19372 & -0.39951 & 0.82169 \\
-0.05472 & -0.07654 & -0.68142 & -0.33994 \\
0.43503 & -0.01918 & -0.05231 & 0.23509 \\
0.09694 & 0.03181 & -0.84492 & 0.04697 \\
-0.12708 & 0.55181 & -0.06748 & 0.23016 \\
-0.12621 & 0.38569 & 0.27805 & 0.36932 \\
\hline
\end{tabular}

Table 4 it shows different decomposition wavelet coefficients of different components. The risk neutral MGF given as the sum of the wavelet coefficients is 2.440658 . This MGF is plugged into the wavelet formula to get the value of a European call option. After the transformation and the decomposition of wavelet coefficients was done, thresholding was performed. The following threshold values were obtained; 2.026467, 4.517850, 5.404695, 7.529094, $9.361670,9.721155$ and 9.038083 . Figure 3 shows how the wavelet decomposition of stock prices is d into 4 frequency bands.

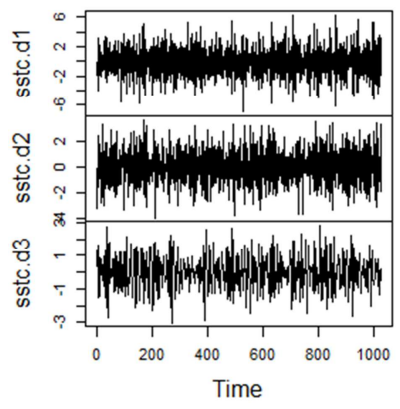

simmstock

Figure 3. Wavelet decomposed results of stock prices into 4 frequency bands.
Figure 4, shows the wavelet decomposition coefficients where we have the filter type being Haar wavelet, the type of decomposition is wavelet and the type of boundary handling is periodic. Clearly, it can be noted that as the resolution level becomes higher, the scales becomes finer, as the coefficients gets smaller.

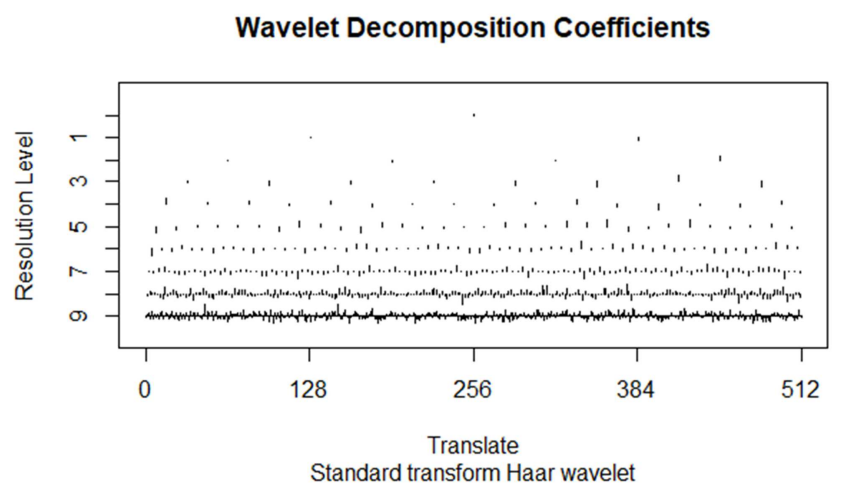

Figure 4. Wavelet Decomposition Coefficients.

From the wavelet -based pricing model equation, the current stock price $(S 0)$ is 100 . Therefore, the values of European call options acquired from the estimated riskneutral MGF are shown table 5.

Table 5. Value of European call option using Wavelet Based Pricing model with period to matureness of 1 year and 2 years.

\begin{tabular}{lll}
\hline Strike Price & Call option value (1 year) & Call option value (2 years) \\
\hline 90 & 21.56864 & 23.96375 \\
91 & 19.52111 & 21.68821 \\
92 & 17.44859 & 19.38615 \\
93 & 15.35135 & 17.05602 \\
94 & 13.22965 & 14.69872 \\
95 & 11.08375 & 12.31453 \\
96 & 8.91390 & 9.90373 \\
97 & 6.72036 & 7.46601 \\
98 & 4.50337 & 5.00344 \\
99 & 2.26317 & 2.51448 \\
100 & 0.00001 & 0.00002 \\
\hline
\end{tabular}

Comparing table 1 and 5, the wavelet values are greater than the Black-Scholes values. In table 5, it can also be noted that as the strike price becomes larger, the value of call option becomes smaller. it can be noted that when the period to matureness is 2 years the value of a call option is also slightly higher than when the period to matureness is 1 year.

This can be illustrated more in figure 5, where it can be seen that the value of a call option is higher when the time to maturity is 2 years than when the time to maturity is 1 year. 
From figure 5, it can also be noted that as the exercise price increases, the value of European call option value decreases.

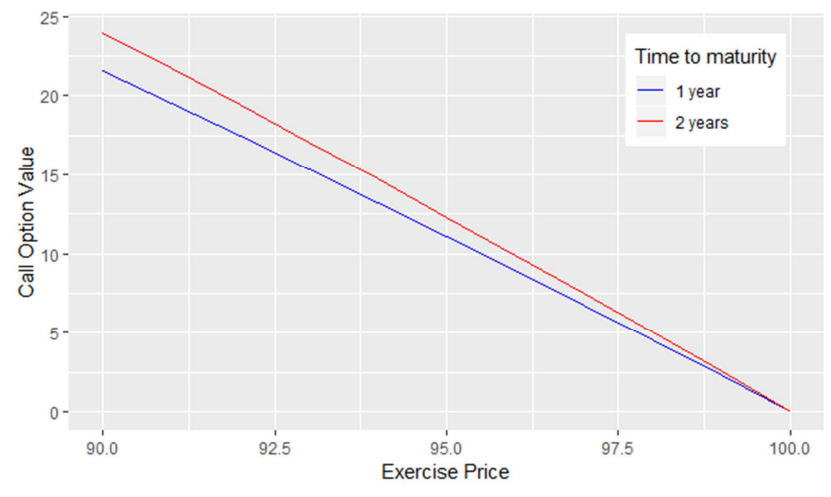

Figure 5. Exercise price against Wavelet call option value.

\section{Pricing performance of the models}

During this study, the MSE and the RMSE were used as the measures of pricing accuracy of the in-sample performance of the two models. MSE and RMSE are chosen as the measures of fit because Wavelet model are estimated by using the SSE. Since the data is divided into 3, the performance comparison is done according to the data division. That is, OTM, ITM, ATM and overall options. The results of the price comparison are shown in table 6 .

Table 6. A Summary of price comparison statistics of different models.

\begin{tabular}{llll}
\hline Options & Statistic & BS & Wavelet \\
\hline \multirow{2}{*}{ OTM } & MSE & 0.884678 & 0.102894 \\
& RMSE & 0.940573 & 0.320771 \\
ITM & MSE & 0.643467 & 0.376454 \\
& RMSE & 0.803164 & 0.613558 \\
ALL & MSE & 0.854388 & 0.208546 \\
& RMSE & 0.924331 & 0.456669 \\
\hline
\end{tabular}

From table 6, it can be seen that wavelet models underpriced the ITM options while BS model overpriced them more. It is also observed that, OTM options are slightly overpriced by the Wavelet model and underpriced by BlackScholes model.

Comparing ALL options, the Black-Scholes estimates shows a larger bias as compared to Wavelet model with a smaller bias. For all the types of options, it can be observed that RMSE and MSE of Wavelet model are lower than those of Black-Scholes model.

In conclusion, it is known that for MSE and RMSE the smaller the values the better the model that is lower values indicates a better fit and therefore in this case Wavelet model performs better than the Black-Scholes model.

\section{Conclusion}

The focus on this study was on pricing of a European Call option using two models, one parametric model (BS Model) and one non-parametric models (Wavelet Pricing Model). After valuation of the option was done, we compared the pricing performance of the three models.
In order to estimate the risk neutral Moment Generating Function of the underlying security from option prices, we applied the methodology of wavelet. Therefore, one of the most significant input of this study is that the wavelet -based pricing model is an alternative model for pricing options and other derivatives on the same underlying asset with varying times to maturity and different strike values. The superiority of the wavelet method comes from the ability of the wavelets to estimate the risk neutral MGF. This is evident from the values of the RMSE and MSE, whereby MSE of wavelet model is lower than that of Black-Scholes model.

We recommend more further investigation on the nonparametric models since most of the studies have focused on the parametric model especially Black-Scholes model. A lot of focus should be put on the Wavelet model because it is a new method in the field of finance.

In this study, we also focused on pricing European call options and therefore we recommend an extension of the approach to pricing more complex options like American options which have no general closed form analytical solution. Other complex options include; Bermuda options and exotic options. Moreover, hedging performance may be evaluated with respect to each of the two models.

\section{References}

[1] P. Boyle, M. Broadie, and P. Glasserman, "Monte Carlo methods for security pricing," J. Econ. Dyn. Control, vol. 21, no. 8-9, pp. 1267-1321, 1997.

[2] F. Black and M. Scholes, "The pricing of options and corporate liabilities," J. Polit. Econ., vol. 81, no. 3, pp. 637654, 1973.

[3] D. S. Bates, "Post-' 87 crash fears in the S\&P 500 futures option market," J. Econom., vol. 94, no. 1-2, pp. 181-238, 2000 .

[4] C. Ma, Advanced asset pricing theory, vol. 2. World Scientific, 2011.

[5] R. Garcia and R. Gençay, "Pricing and hedging derivative securities with neural networks and a homogeneity hint," $J$. Econom., vol. 94, no. 1-2, pp. 93-115, 2000.

[6] E. Haven, X. Liu, C. Ma, and L. Shen, "Revealing the implied risk-neutral MGF from options: The wavelet method," $J$. Econ. Dyn. Control, vol. 33, no. 3, pp. 692-709, 2009.

[7] E. Haven, X. Liu, and L. Shen, "De-noising option prices with the wavelet method," Eur. J. Oper. Res., vol. 222, no. 1, pp. 104-112, 2012.

[8] J. B. Ramsey and C. Lampart, "Decomposition of economic relationships by timescale using wavelets," Macroecon. Dyn., vol. 2, no. 1, pp. 49-71, 1998.

[9] R. Weron, "Heavy-tails and regime-switching in electricity prices," Math. Methods Oper. Res., vol. 69, no. 3, pp. 457473, 2009.

[10] E. Capobianco, "Wavelet transforms for the statistical analysis of returns generating stochastic processes," Int. J. Theor. Appl. Financ., vol. 4, no. 03, pp. 511-534, 2001. 
[11] E. W. Sun and T. Meinl, "A new wavelet-based denoising algorithm for high-frequency financial data mining," Eur. $J$. Oper. Res., vol. 217, no. 3, pp. 589-599, 2012.

[12] H. Asgharian, "A conditional asset-pricing model with the optimal orthogonal portfolio," J. Bank. Financ., vol. 35, no. 5, pp. 1027-1040, 2011.

[13] A.-M. Matache, P.-A. Nitsche, and C. Schwab, "Wavelet Galerkin pricing of American options on Lévy driven assets," Quant. Financ., vol. 5, no. 4, pp. 403-424, 2005.
[14] L. Ortiz-Gracia and C. W. Oosterlee, "A highly efficient Shannon wavelet inverse Fourier technique for pricing European options," SIAM J. Sci. Comput., vol. 38, no. 1, pp. B118--B143, 2016.

[15] V. Genon-Catalot, C. Laredo, and D. Picard, "Non-parametric estimation of the diffusion coefficient by wavelets methods," Scand. J. Stat., pp. 317-335, 1992.

[16] M. J. Jensen, "Using wavelets to obtain a consistent ordinary least squares estimator of the long-memory parameter," $J$. Forecast., vol. 18, no. 1, pp. 17-32, 1999. 